\section{As redes de atenção à saúde}

\section{H ealth care networks}

Eugênio Vilaça M endes ${ }^{1}$
${ }^{1}$ Secretaria de Estado de Saúdede M inas Gerais. Rua Sapucai 429/10 andar, Floresta. 30150-050 Belo Horizonte M G.

eugeniov@saude.mg.gov.br
Abstract The demographic and epidemiologic transition resulting from aging and the increase of life expectation means an increment related to chronic conditions. The healthcare systems contemporary crisis is characterized by the organization of the focus on fragmented systems turned to theacute conditions care, in spite of thechronic conditions prevalence, and by the hierarchical structure without communication flow among the different health care levels. Brazil health care situation profile is now presenting a triple burden of diseases, due to the concomitant presence of infectious diseases, external causes and chronic diseases. The solution is to restore the consistence between thetripleburden of diseases on the health situation and the current system of healthcare practice, with the implantation of health care networks. The conclusion is that there are evidences in the international literature on health care networks that these networks may improve the clinical quality, the sanitation results and the user's satisfaction and the reduction of healthcare systems costs.

Key words Comprehensive health care, Demographic transition, Health transition, Chronic disease
Resumo Astransições demográfica eepidemiológica significam um incremento relativo das condições crônicas. Tem sido assim, no Brasil, que apresenta uma situação de saúde detripla carga de doenças, manifestada na convivência de doenças infecciosas, parasitárias e problemas de saúde re produtiva, causas externas e doenças crônicas. Há uma crise dos sistemas de saúde contemporâneos que se explica pela incoerência entre uma situação de saúde com predomínio relativo forte de condições crônicas e uma resposta social através de sistemas fragmentados e voltados, principalmente, para as condi ções agudas eas agu dizações das condições crônicas. Essa crise se manifesta em nosso país, tanto no setor público quanto no setor privado. A solução para essa crise está em recompor a coerência entrea situação de tripla carga de doenças com uma resposta social estruturada em sistemasintegrados de saúde: as redes de atenção à saúde. Conclui-se que há evidências na literatura internacional de que as redes de atenção à saúde podem melhorar a qualidade dos serviços, os resultados sanitários e a satisfação dos usuários e reduzir os custos dos sistemas de atenção à saúde. Palavras-chave Assistência integral à saúde, Transição demográfica, Transição epidemiológica, Doença crônica 


\section{A situação de saúde no Brasil}

Os sistemas de atenção à saúde são respostas sociais deliberadas às necessidades de saúde da população. Assim, ao se discutir uma proposta de organização do Sistema Único de Saúde (SUS), deve-se começar por analisar que necessidades de saúde se expressam na população brasileira. A situação de saúde dos brasileiros é analisada nos seus aspectos demográficos e epidemiológicos.

Do ponto de vista demográfico, o Brasil vive uma transição demográfica acelerada. A população brasileira, apesar de baixas taxas de fecundidade, vai continuar crescendo nas próximas dé cadas, como resultado dos padrões de fecundidade anteriores. 0 percentual de pessoas idosas maiores de 65 anos, que era de 2,7\% em 1960, passou para 5,4\% em 2000 e alcançará 19\% em 2050, superando o número de jovens ${ }^{1}$.

Uma população em processo rápido de enveIhecimento significa um crescenteincremento relativo das condições crônicas por que essas condições de saúde afetam mais os segmentos de maior idade. Os dados da Pesquisa Nacional de Amostra Domiciliar do IBGE de 2008 mostram que $79,1 \%$ dos brasileiros de mais de 65 anos de idade relataram ser portadores de, pelo menos, uma das doze doenças crônicas selecionadas².

Por outro lado, na perspectiva epidemiológi$\mathrm{ca}$, o país vivencia uma forma detransição singular, diferente da transição clássica dos países desenvolvidos. Para a análise epidemiológica, vai se considerar o conceito decondições de saúde, agudas e crônicas, que difere da tipologia mais usual que é doenças transmissíveis e doenças e agravos não transmissíveis. Essa tipologia convencional tem sido muito útil nos estudos epidemiológicos, mas observa-se que ela não se presta para referenciar a estruturação dos sistemas de atenção à saúde. Para organizar os sistemas de atenção à saúde, o mais conveniente é separar as condições agudas, em geral de curso curto e que podem ser respondidas por um sistema reativo e com respostas episódicas, das condições crônicas, quetêm curso mais ou menos longo eque exigem um sistema que responda a elas de forma proativa, contínua e integrada. Por isso, as condições crônicas envolvem todas as doenças crônicas, mais as doenças transmissíveis de curso longo (tuberculose, hanseníase, HIV/aids e outras) , as condições maternas e infantis, os acompanhamentos por ciclos de vida (puericultura, herbicultura e seguimento das pessoas idosas), as deficiências físicas e estruturais contínuas (amputações, cegueiras e deficiências motoras persistentes) e os distúrbios mentais de longo prazo ${ }^{3,4}$.
Uma análise da mortalidade no Brasil indica que, em 1930, as doenças infecciosas respondiam por $46 \%$ das mortes, mas que este valor decresceu para um valor próximo a 5\% em 2000; ao mesmo tempo, as doenças cardiovasculares, que representavam em torno de $12 \%$ das mortes em 1930, responderam, em 2000, por quase $30 \%$ de todos os óbitos ${ }^{5}$. Em relação à morbidade, medida pela morbidade hospitalar, no ano de 2005, das primeiras quinze causas de internações pelo SUS, nove foram por condições crônicas ${ }^{6}$.

A análise da carga de doenças, medida em anos de vida perdidos ajustados por incapacidade, demonstra que $14,7 \%$ dessa carga são por doenças infecciosas, parasitárias e desnutrição; 10,2\%, por causas externas; 8,8\%, por condições maternas e perinatais e $66,3 \%$, por doenças crônicas ${ }^{7}$. O somatório das duas últimas, ambas condições crônicas, indica que $75 \%$ da carga de doenças no país são determinados por condições crônicas, o que, ainda, exclui o percentual de doenças transmissíveis de curso longo.

A situação epidemiológica brasileira distancia-se da transição epidemiológica clássica omramiana ${ }^{8}$, observada nos países desenvolvidos, etem sido definida, recentemente, como tripla carga de doenças por que envolve, ao mesmo tempo, uma agenda não concluída de infecções, desnutrição e problemas de saúde reprodutiva; o desafio das doenças crônicas e deseus fatores de riscos, como o tabagismo, o sobrepeso, a obesidade, a inatividade física, o estresse e a alimentação inadequada; e o forte crescimento das causas externas 9 .

\section{O problema fundamental dos sistemas de atenção à saúde contemporâneos}

A crise contemporânea dos sistemas de aten ção à saúde que se manifesta, em maior ou menor grau, em todos os países mundo, decorre deuma incoerência entre uma situação de saúde detransição demográfica e de transição epidemiológica completa nos países desenvolvidos e de dupla ou tripla carga de doenças nos países em desenvolvimento e o modo como se estruturam as respostas sociais deliberadas às necessidades das populações.

A situação de saúde de forte predomínio relativo das condições crônicas não pode ser respondida, com eficiência, efetividade equalidade, por sistemas de saúde voltados, prioritariamente, para as condições agudas e para as agudizações de condições crônicas, eorganizados de forma fragmentada. 
Essa crise decorre da incongruência entre uma situação de saúde do século XXI, convivendo com um sistema de atenção à saúde do século XX. Isso não deu certo nos países desenvolvidos e isso não está dando certo no Brasil, nem no setor público, nem no setor privado.

$\mathrm{Ham}^{10}$ faz um diagnóstico dessa crise a partir de uma análise histórica dos sistemas de atenção à saúde, mostrando que, até a primeira metade do século XX, eles se voltaram para as doenças infecciosas e, na segunda metade daquel e século, para as condições agudas e para as agudizações das doenças crônicas. E ressalta: 0 paradigma predominante da condição aguda éum anacronismo. Ele foi formatado pela noção do século XIX da doença como ruptura de um estado normal determinada por um agente externo ou por um trauma. Sob esse modelo a atenção, a condição aguda é o que representa, diretamente, a ameaça. M asa epidemiologia moderna mostra que os problemas de saúde prevalecentes hoje, definidos em termos de impactos sanitários e econômicos, gi ram em torno das condições crônicas. Na mesma linha, a O rganização Mundial da Saúde ${ }^{4}$ adverte de forma incisiva: Ossistemas de saúdepredominantes em todo mundo estão falhando, pois não estão conseguindo acompanhar a tendência de declínio dos problemas agudos e de ascensão das condições crônicas. Quando os problemas de saúde são crônicos, 0 modelo de tratamento agudo não funciona.

Uma explicação para essa crise é feita por Bengoa ${ }^{11}$, quando assinala que os sistemas de atenção à saúde movem-se numa relação dialética entrefatores contextuais como envel hecimento da população, transição epidemiológica e inovação tecnológica e fatores internos como cultura organizacional, recursos institucionais, sistemas de incentivo, estrutura organizacional eestilos de liderança e gestão. Os fatores contextuais, externos aos sistemas de atenção à saúde, mudam em ritmos mais rápidos que os fatores internos, os que estão sob a governabilidade setorial. Isso faz com que os sistemas de atenção à saúde não tenham a capacidade de adaptar-se, oportunamente, às mudanças contextuais. Nisso reside a crise universal dos sistemas de atenção à saúde que foram concebidos e desenvolvidos com uma presunção de continuidade de uma atuação voltada para as condições e eventos agudos, desconsiderando a epidemia moderna das condições crônicas.

A transição da situação de saúde, juntamente com outros fatores como o desenvolvimento científico, tecnológico e econômico, determina a transição da atenção à saúde. Por essa razão, em qualquer tempo e em qualquer sociedade, deve haver uma coerência entre a situação de saúde e o sistema de atenção à saúde. Quando essa coerência se rompe, como ocorre, neste momento, em escala global e no Brasil, instala-se uma crise nos sistemas de atenção à saúde.

\section{Ossistemasfragmentados}

de atenção à saúde

Uma análise dos sistemas de atenção à saúde, feita numa perspectiva internacional, mostraque eles são dominados pelos sistemas fragmentados, voltados para atenção às condições agudas e às agudizações de condições crônicas.

Conceitualmente, os sistemas fragmentados de atenção à saúde são aqueles que se organizam através de um conjunto de pontos de atenção à saúde isolados e incomunicados uns dos outros e que, por consequência, são incapazes de prestar uma atenção contínuaà população. Em geral, não há uma população adscrita de responsabilização, o que impossibilita a gestão baseada na população. N eles, a atenção primária à saúde não se comunica fluidamente com a aten ção secundária à saúde e esses dois níveis também não se comunicam com a atenção terciária à saúde, nem com os sistemas de apoio. N esses sistemas, a atenção primária à saúde não pode exercitar seu papel de centro de comunicação, coordenando o cuidado.

Os sistemas fragmentados caracterizam-se pela forma de organização hierárquica; a inexistência da continuidade da atenção; o foco nas condições agudas através de unidades de pronto-atendimento, ambulatorial e hospitalar; a passividade da pessoa usuária; a ação reativa à demanda; a ênfase relativa nas intervenções curativas e reabilitadoras; o modelo de atenção à saúde, fragmentado e sem estratificação dos riscos; a atenção centrada no cuidado profissional, especialmente no médico; e o financiamento por procedimentos ${ }^{12}$.

Os sistemas fragmentados têm sido um desastre sanitário e econômico em todo o mundo.

Tomese o exemplo brasileiro. Pesquisa da Fiocruz da Bahia e da Unifesp, medindo a hemoglobina glicada de 6.700 portadores de diabetes em 22 centros clínicos brasileiros, evidenciou que o nível glicêmico só estava controlado (valor igual ou inferior a $7 \%$ ) em $10 \%$ dos portadores de diabetes tipo 1 eem $25 \%$ dos portadores de diabetes tipo 2. Além disso, verificou que $45 \%$ dos examinados apresentavam sinais de retinopatias, $44 \%$, de neuropatias e $16 \%$, de alterações renais ${ }^{13}$. 
Os resultados do controle do diabetes não são muito melhores nos Estados Unidos. Nesse país, havia oito milhões de portadores de diabe tes e um outro tanto sem diagnósticos. Dos diagnosticados, 30\% não estavam controlados, $35 \%$ desenvolveram nefropatias, $58 \%$, doenças cardiovasculares, 30 a $70 \%$, neuropatias, pouco mais de $50 \%$ realizaram o exame oftalmológico de rotina e a carga econômica da doença foi de noventa bilhões de dólares anuais ${ }^{14}$.

Chama a atenção que, em dois países muito diferentes, os resultados dos desfechos clínicos do diabetes são muito negativos, sendo que 0 Brasil tem um gasto per capita anual de US\$ 427,00 e os Estados Unidos, de US \$ 6.719,00, 0 que sinaliza que o problema do manejo das condições crônicas não está no volume de recursos despendidos, mas na forma como se organizam os sistemas de atenção à saúde ${ }^{6}$.

Recompondo a coerência entre uma situação de saúde de tripla carga de doenças e o sistema deatenção à saúde: as redes deatenção à saúde

A solução do problema fundamental do SUS consiste em restabelecer a coerência entre a situação de saúde de tripla carga de doenças, com predominância relativa forte de condições crônicas, e o sistema de atenção à saúde. I sso vai exigir mudanças profundas que permitam superar 0 sistema fragmentado vigente através da implantação de redes de atenção à saúde.

O conceito de redes de atenção à saúde

As redes de atenção à saúde são organizações poliárquicas de conjuntos de serviços de saúde, vinculados entre si por uma missão única, por objetivos comuns e por uma ação cooperativa e interdependente, que permitem ofertar uma atenção contínua e integral a determinada população, coordenada pela atenção primária à saúde prestada no tempo certo, no lugar certo, com o custo certo, com a qualidade certa e de forma humanizada -, e com responsabilidades sanitárias e econômicas por esta população 6 .

Dessa definição, emergem os conteúdos básicos das redes de atenção à saúde: apresentam missão e objetivos comuns; operam de forma cooperativa e interdependente; intercambiam constantemente seus recursos; são estabelecidas sem hierarquia entre os diferentes componentes, organizando-se de forma poliárquica, em que todos os pontos de atenção à saúde são igualmente importantes e se relacionam horizontalmente; implicam um contínuo de atenção nos níveis primário, secundário e terciário; convocam uma aten ção integral com intervenções promocionais, preventivas, curativas, cuidadoras, reabilitadoras e paliativas; funcionam sob coordenação da atenção primária à saúde; prestam atenção oportuna, em tempos e lugares certos, de forma eficiente e ofertando serviços seguros e efetivos, em consonância com as evidências disponíveis; focam-se no ciclo completo de aten ção a uma condição de saúde; têm responsabilidades sanitárias e econômicas inequívocas por sua população; e geram valor para a sua população.

Da definição operacional de redes adotada, fica claro que ela se aproxima, conceitualmente, da estrutura em redes que implica missão única, objetivos comunse planejamento conjunto eque se distancia da concepção de networking que conota interações informais fortemente impulsionadas pelas tecnologias de informação.

Os elementos constitutivos

das redes de atenção à saúde

As redes de atenção à saúde constituem-se de três elementos: a população, a estrutura operacional e o modelo de atenção à saúde.

\section{A população}

0 primeiro elemento das redes de atenção à saúde e sua razão de ser é uma população, colocada sob sua responsabilidade sanitária e econômica. É isso que marca a atenção à saúde baseada na população, uma característica essencial das redes de atenção à saúde.

A atenção à saúde baseada na população é a habilidade de um sistema em estabelecer as necessidades de saúde de uma população específica, sob sua responsabilidade, segundo os riscos, de implementar e avaliar as intervenções sanitárias relativas a essa população e de prover o cuidado para as pessoas no contexto de sua cultura e de suas preferências ${ }^{15}$.

A população de responsabilidade das redes de atenção à saúde vive em territórios sanitários singulares, organiza-se socialmente em famílias eécadastrada e registrada em subpopulações por riscos sociossanitários. Assim, a população total de responsabilidade de uma rede de atenção à saúde deve ser plenamente conhecida e registrada em sistemas deinformação potentes. M as não basta o conhecimento da população total: ela deve ser segmentada, subdividida em subpopulações 
por fatores de riscos e estratificada por riscos em relação às condições de saúde estabelecidas. 0 conhecimento da população deuma rededeatenção à saúde envolve um processo complexo, estruturado em vários momentos, sob a responsabilidade fundamental da atenção primária: 0 processo de territorialização; o cadastramento das famílias; a classificação das famílias por riscos sociossanitários; a vinculação das famílias à unidade de atenção primária à saúde/equipe do Programa de Saúde da Família; a identificação desubpopulações com fatores de riscos; a identificação das subpopulações com condições de saúde estabelecidas por graus de riscos; e a identificação de subpopulações com condições de saúde muito complexas.

A estrutura operacional

O segundo elemento constitutivo das redes de atenção à saúde é a estrutura operacional, constituída pel os nós das redes e pel as ligações materiais eimateriais que comunicam esses diferentes nós.

A estrutura operacional das redes de atenção à saúde compõe-se decinco componentes: o centro de comunicação, a atenção primária à saúde; os pontos de aten ção secundários e terciários; os sistemas de apoio; os sistemas logísticos; e o sistema de governança da rede de atenção à saúde. Os três primeiros correspondem aos nós das redes e o quarto, às ligações que comunicam os diferentes nós. E o quinto, o componente que governa as relações entre os quatro primeiros.

0 centro de comunicação das redes de atenção à saúde é o nó intercambiador no qual se coordenam os fluxos e contrafluxos do sistema de atenção à saúde e é constituído pela atenção primária à saúde (unidade de atenção primáriaà saúde ou equipe do Programa de Saúde da Família).

Há evidências de que os sistemas de aten ção à saúde baseados numa forte orientação para a atenção primária à saúde, contrastados com os sistemas de baixa orientação para a aten ção primária à saúde, são mais adequados por que se organizam a partir das necessidades de saúde da população; mais efetivos porque são a única forma de enfrentar consequentemente a situação epidemiológica de hegemonia das condições crônicas e por impactar significativamente os níveis de saúde da população; mais eficientes por que apresentam menores custos e reduzem procedimentos mais caros; mais equitativos por que discriminam positivamente grupos e regiões mais pobres ediminuem o gasto do bolso das pessoas efamílias; edemaior qualidade por que colocam ênfase na promoção da saúde e na prevenção das doenças e ofertam tecnologias mais seguras para os usuários e profissionais de saúde ${ }^{16-20}$.

Contudo, para que a atenção primária à saúde possa resultar em todos esses benefícios, deve ser reformulada para cumprir três papéis essenciais nas redes de atenção à saúde: a resolução, a capacidade para solucionar mais de $85 \%$ dos problemas de saúde de sua população; a coordenação, a capacidade de orientar os fluxos e contrafluxos de pessoas, informações e produtos entre os componentes das redes; e a responsabilização, a capacidade de acolher e responsabilizar-se, sanitária e economicamente, por sua população.

O segundo componente das redes de atenção à saúde são os pontos de atenção secundários e terciários, os nós das redes onde se ofertam determinados serviços especial izados, gerados através de uma função de produção singular. Eles se diferenciam por suas respectivas densidades tecnológicas, sendo os pontos de atenção terciários mais densos tecnologicamente que os pontos de atenção secundários e, por essa razão, tendem a ser mais concentrados espacialmente.

Contudo, na perspectiva das redes poliárquicas, não há, entre eles, relações de principalidade ou subordinação, características das relações hierárquicas, já que todos são igualmente importantes para seatingirem os objetivos comuns das redes de atenção à saúde.

0 terceiro componente das redes de atenção à saúde são os sistemas de apoio. Os sistemas de apoio são os lugares institucionais das redes onde se prestam serviços comuns a todos os pontos de atenção à saúde, nos campos do apoio diagnóstico e terapêutico, da assistência farmacêutica e dos sistemas de informação em saúde.

O sistema de apoio diagnóstico e terapêutico envolve os serviços de diagnóstico por imagem, os serviços de medicina nuclear diagnóstica e terapêutica, a eletrofisiologia diagnóstica eterapêutica, as endoscopias, a hemodinâmica ea patologia clínica (anatomia patológica, genética, bioquímica, hematologia, imunologia e microbiologia e parasitologia).

0 sistema de assistênciafarmacêutica envolve uma organização complexa, exercitada por um grupo de atividades relacionadas com os medicamentos, destinadas a apoiar as ações de saúde demandadas por uma população, englobando intervenções logísticas relativas à seleção dos medicamentos, à programação de medicamentos, à aquisição de medicamentos, ao armazenamento dos medicamentos, à distribuição dos medicamentos, bem como ações assistenciais da farmácia clínica, como o formulário terapêutico, a dis- 
pensação, a adesão ao tratamento, a conciliação de medicamentos e a farmacovigilância.

A construção social das redes de atenção à saúde, para ser consequente, tem de ser suportada por informações de qualidade, ofertadas por bons sistemas de informação em saúde. Os sistemas de informação em saúde compreendem os determinantes sociais da saúde e os ambientes contextuais elegais nos quais os sistemas deatenção à saúde operam; os insumos dos sistemas de atenção à saúde e os processos relacionados a eles, incluindo a política e a organização, a infraestrutura sanitária, os recursos humanos e os recursos financeiros; a performance dos sistemas de atenção à saúde; os resultados produzidos em termos de mortalidade, morbidade, carga de doenças, bem-estar e estado de saúde; e a equidade em saúde.

0 quarto componente das redes de atenção à saúde são os sistemas logísticos. Os sistemas logísticos são soluções tecnológicas, fortemente ancoradas nas tecnologias de informação, que garantem uma organização racional dos fluxose contrafluxos de informações, produtos e pessoas nas redes de atenção à saúde, permitindo um sistema eficaz de referência e contrarreferência das pessoas e trocas eficientes de produtos e informações, ao longo dos pontos de atenção à saúde e dos sistemas de apoio, nas redes de atenção à saúde. Os principais sistemas logísticos das redes de atenção à saúde são o cartão de identificação das pessoas usuárias, o prontuário clínico, os sistemas de acesso regulado à atenção à saúde e os sistemas de transporte em saúde.

0 quinto componente das redes de atenção à saúde são os sistemas de governança. A governança das redes de atenção à saúde é o arranjo organizativo que permite a gestão de todos os componentes das redes de atenção à saúde, de forma a gerar um excedente cooperativo entre os atores sociais em situação, aumentar a interdependência entre eles e obter resultados sanitários e econômicos para a população adscrita. A governança objetiva criar uma missão e uma visão nas organizações; definir objetivos e metas que devem ser alcançados no curto, médio e longo prazos para cumprir com a missão e a com visão; articular as políticas institucionais para 0 cumprimento dos objetivos e metas; e desenvolver a capacidade de gestão necessária para planejar, monitorar e avaliar o desempenho dos gerentes e da organização. A governança das redes de atenção à saúde, no SUS, deve ser feita por meio dearranjos interfederativos, coerentes com o federalismo cooperativo que se pratica no Bra- sil. São as comissões intergestores que se materializam: no plano nacional, na comissão intergestores tripartite; nos estados, nas comissões intergestores bipartite; e nas regiões de saúde, nas comissões intergestores bipartite regionais.

0 modelo de atenção à saúde

0 terceiro elemento constitutivo das redes de atenção à saúde são os modelos de atenção à saúde.

Os modelos de aten ção à saúde são sistemas lógicos que organizam o funcionamento das redes de atenção à saúde, articulando, de forma singular, as relações entre a população e suas subpopulações estratificadas por riscos, os focos das intervenções do sistema de atenção à saúdee os diferentes tipos de intervenções sanitárias, definidos em função da visão prevalecente da saúde, das situações demográfica e epidemiológica e dos determinantes sociais da saúde, vigentes em determinado tempo e em determinada sociedade. A necessidade de se mudarem os sistemas de atenção à saúde para que possam responder com efetividade, eficiência e segurança a situações de saúde dominadas pelas condições crônicas levou ao desenvolvimento dos modelos de atenção à saúde. Há modelos de atenção à saúde para as condições agu das e crônicas.

As condições agudas e os eventos agudos decorrentes de condições crônicas agudizadas exigem, para o seu manejo adequado, a implantação de modelos de aten ção à saúde que, em geral, expressam-senum tipo de classificação de riscos. Isso se deve a que, nas condições agudas, a variável-chave para a organização das redes de atenção às urgências eàs emergências éo tempo-resposta em relação ao risco. Os modelos de triagem nas urgências e emergências mais avançados e que foram construídos numa concepção sistêmica são o modelo australiano, o modelo pioneiro que usa tempos de espera de acordo com a gravidade; o modelo canadense, semeIhante, mas mais complexo que o australiano; 0 modelo americano, que opera com um único algoritmo e que se foca mais na necessidade de recursos para o atendimento; o modelo de Andorra, que se articula em sintomas, discriminantes e algoritmos, mas muito complexo e demorado; e o sistema de triagem de $M$ anchester, que opera com algoritmos e determinantes, associado a tempos de espera simbolizados por cinco cores e que tem sido usado em vários países ${ }^{21}$.

Por outro lado, os modelos de atenção à saúde, destinados à orientação dos sistemas de atenção à saúde, voltados para as condições crôni- 
cas, são construídos a partir de um modelo se minal, o modelo de atenção crônica, o M AC 22 . Dele, derivam várias adaptações aplicadas em diferentes partes do mundo, tanto em países desenvolvidos como em países em desenvolvimento. Ele tem sido adotado, com modificações adjetivas, no Canadá, Reino Unido, Alemanha, Rússia, Espanha, Austrália, Dinamarca, Holanda e em alguns países em desenvolvimento ${ }^{4,23-27}$. No Brasil, M endes ${ }^{6}$ propôs, também com base no M AC, um modelo de atenção às condições crônicas para utilização no SUS.

O M AC compõe-se de seis el ementos, subdivididos em dois grandes campos: 0 sistema de atenção à saúde e a comunidade. No sistema de atenção à saúde, as mudanças devem ser feitas na organização da atenção à saúde, no desenho do sistema de prestação de serviços, no apoio às decisões, nos sistemas de informação clínica eno autocuidado apoiado. $\mathrm{Na}$ comunidade, as mudanças estão centradas na articulação dos serviços de saúde com os recursos da comunidade. Esses seis elementos apresentam interrelações que permitem desenvolver usuários informados e ativos e equipe de saúde preparada e proativa para produzir melhores resultados sanitários e funcionais para a população.

Há evidências abundantes e robustas, na literatura internacional, sobre os efeitos positivos do M AC, seja na sua avaliação conjunta, seja na avaliação de seus elementos separadamente. 0 estudo avaliativo clássico desse modelo foi realizado pela Rand Corporation e pela Universidade de Berkeley ${ }^{28}$ e teve dois objetivos: avaliar as mudanças ocorridas nas organizações de saúde para implementar o M AC e estabelecer o grau em que a adoção deste modelo melhora os processos e os resultados em relação às condições crônicas. Esta avaliação durou quatro anos e envolveu aproximadamente quatro mil portadores de diabetes, insuficiência cardíaca, asma e depressão, em 51 organizações de saúde e gerou uma grandequantidade de publicações que mostram que o model o funciona. Vários outros trabalhos de avaliação do MAC estão disponíveis na literatura. Alguns são de avaliação geral da aplicação do modelo ${ }^{10,29,30}$, mas há trabal hos que avaliam a melhoria da qualidade dos serviços de atenção às condições crônicas ${ }^{31}$; condições crônicas particulares ${ }^{32}$; aspectos organizacionais ${ }^{33} \mathrm{e}$ avaliação econômica ${ }^{34}$.
As evidências

sobre as redes de atenção à saúde

Há, na literatura internacional, provinda de vários países, evidências de boa qualidade dequeas redes de aten ção à saúde podem melhorar a qualidade clínica, os resultados sanitários, a satisfação dos usuários e reduzir os custos dos sistemas de atenção à saúde.

Esses resultados foram positivos em várias situações: na atenção às pessoas idosas ${ }^{35,36 ;}$ na saúde mental ${ }^{37-39}$; no controle do diabete ${ }^{40-42}$; no aumento da satisfação dos usuários ${ }^{43,44}$; no controle de doenças cardiovasculares ${ }^{45}$; no controle de doenças respiratórias crônicas ${ }^{46}$ e na redução da utilização de serviços especializados ${ }^{29}$.

Uma avaliação de 72 sistemas que utilizaram al guma forma deintegração concluiu que os programas queintegravam a atenção primária à saúde com os outros níveis eque tinham uma população adscrita foram mais efetivos eque seus usuários estavam mais satisfeito ${ }^{43}$. Um ensaio randomizado verificou quea integração entrea atenção primária e especializada à saúde permitiu a identificação de pessoas com alto risco de hospitalização e reduziu o uso de serviços especializados $^{44}$. Uma análise de catorze revisões sistemáticas e 29 ensaios randomizados encontrou evidências de que as redes de atenção à saúde meIhoraram o uso dos recursos e al guns resultados clínicos selecionados e reduziram os custos da atenção ${ }^{29}$. $\mathrm{Na}$ Espanha, concluiu-se que existem evidências sólidas de que os enfoques e intervenções dos sistemas integrados mostraram resultados positivos em vários âmbitos e patologi$a s^{25,47}$. Há evidências de que a integração de gestores e prestadores de serviços melhorou a cooperação entreeles, deu uma maior atenção à gestão de caso, incentivou a utilização de tecnologia de informação e teve algum impacto sobre os custos da atenção à saúde ${ }^{48}$.

No Brasil, o tema das redes de atenção à saúde érecente enão há experiências em escala, nem avaliações robustas. Contudo, estudos de casos de experiências de redes de atenção à saúde indicam que elas, à semelhança do que ocorre em países desenvolvidos, podem ter impacto significativo nos níveis de saúde, com custos suportáveis pelo SU ${ }^{49,50}$. 


\section{Referências}

1. Brito F. A transição demográfica no Brasil: as possibilidades e o desafio para a economia e a sociedade. Belo H orizonte: CEDEPLAR/UFM G; 2007.

2. Instituto Brasileiro de Geografia e Estatísticas. U m panorama da saúde no Brasil: acesso e utilização dos serviços, condições de saúde e fatores de risco e proteção da saúde. Rio de Janeiro: IBGE; 2010.

3. von Korff M, Gruman J, Schaefer J, Curry SJ, Wagner $\mathrm{EH}$. Collaborative management of chronic illness. Ann Intern Med 1997; 127:1097-1102.

4. Organização Mundial da Saúde. Cuidados inovadores para condições crônicas: componentes estruturais de ação. Braślia: Organização M undial da Saúde; 2003.

5. Silva JB, Gomes FBC, Cezário AC, M oura L. Doenças e agravos não transmissíveis: bases epidemiológicas. In: Rouquayrol MZ, Almeida Filho N. Epidemiologia e saúde. 6a ed. Rio de Janeiro: M edsi; 2006.

6. Mendes EV. As redes de atenção à saúde. Belo Horizonte: Escola de Saúde Pública de Minas Gerais; 2009.

7. Shramm JMA, Oliveira AF, Leite IC, Valente JG, Gadelha AMJ, Portela M C, Campos M R. Transição epidemiológica e o estudo de carga de doença no Brasil. Cien Saude Colet 2004; 9:987-908.

8. Omran AR. The epidemiologic transition: a theory of the epidemiology of population change. M ilbank M em. Fund 1971; 49:509-583.

9. Frenk J. Bridging the divide: comprehensive reform to improve health in M exico. Nairobi: Commission on Social Determinants of Health; 2006.

10. Ham C. Evaluations and impact of disease manage ment programmes. Bonn: Conference of Bonn; 2007.

11. Bengoa R. Empatanados. Revista de Innovación Sanitaria y Atención Integrada 2008; 1:1.

12. Fernandez JMD. Los sistemas integrados de salud: un modelo para avanzar tras completar las transferencias. Barcelona: B \& F Gestión y Salud; 2004.

13. Dominguez BC. Controle é baixo no Brasil. RADIS 2007; 59:11.

14. Barr CE. Disease state considerations. In: Todd WE, Nash D. Disease management: a system approach to improving patients' outcomes. Chicago: American Hospital Publishing Inc; 1996.

15. Tufts Managed Care Institute. Population-based health care: definitions and applications. [site da Internet] [acessado 2008 set 15]. Disponível em: http:// www.thci.org

16. Starfield B, Shi L. Policy relevant determinants of health: an international perspective. Health Policy 2002; 60:201-216.
17. Macinko J, Starfield B, Shi L. The contribution of primary care systems to health outcomes within OECD countries, 1970-1998. H ealth Serv Res 2003; 38:819-853.

18. Pan American Health Organization. Renewing primary health care in the Americas: a position paper of the Pan American Health Organization. Washington, D.C.: PAH 0; 2005

19. M endes EV. Revisão bibliográfica sobre a atenção primária à saúde. Belo Horizonte: Secretaria de Estado de Saúde de M inas Gerais; 2005.

20. Organização Mundial da Saúde. Relatório M undial de Saúde 2008: A atenção primária à saúde: agora mais do que nunca. Brasília: OM S; 2008.

21. Cordeiro Júnior W. A gestão de riscos na urgência. Belo Horizonte: Grupo Brasileiro de Classificação de Risco; 2008.

22. Wagner EH. Chronic disease management: what will take to improve care for chronic illness? Effective Clinical Practice 1998; 1:2-4.

23. Government of British Columbia. British Columbia expanded chronic care model. [site da Internet] [acessado 2008 out 24]. Disponível em: http://www. health.gov.bc.ca

24. Porter M, Kellogg M. Kaiser Permanente: an integrated health care experience. Revista de Innovación Sanitaria y Atención Integrada 2008; 1:1.

25. Vazquez MLN, Vargas IL. Organizaciones sanitarias integradas: un estudio de casos. Barcelona: Consorci Hospitalari de Catalunya; 2007.

26. Temminkc D, Hutten JB, Francke AL, Rasker JJ, Abu-Saad H H, van der Zee J. Rheumatology outpatient nurse clinics: a valuable addition? Arthritis Rheum. 2001; 45:280-286.

27. Department of Health. Supporting people with longterm conditions: a NHS and social care model to support local innovation and integration. Leeds: Long Term Conditions Team Primary Care/Department of Health; 2005.

28. Rand Health. Improving chronic illness care evaluation: Rand $\mathrm{H}$ ealth Project. [site da Internet] [acessado 2008 set 13]. Disponível em: http://www.rand. org/health/projects

29. Singh D. Transforming chronic care: evidence about improving care for people with long-term conditions. Birmingham: University of Birmingham/ $\mathrm{H}$ ealth Services M anagement Centre; 2005.

30. Singh D, Ham C. Improving care for people with long term conditions: a review of UK and international frameworks. Birmingham: Institute for Innovation and Improvement of University of Birmingham; 2006. 
31. Nutting PA, Dickinson WP, Dickinson LM, Nelson CC, King DK, Crabtree BF, Glasgow RE. Use of chronic care model elements is associated with higher-quality care. Ann Fam M ed 2007; 5:14-20.

32. Ouwens $M$, Wollersheim $H$, Hermens $R$, Hulscher $M$, Grol R. Integrated care programmes for chronically ill patients: a review of systematic reviews. Int. J. Qual. Health Care 2005; 17:141-46.

33. Hung DY, Rundall TG, Crabtree BF, Tallia AF, Cohen DJ, Halpin HA. Influence of primary care practice and provider attributes on preventive service delivery. Am J Prev Med. 2006; 30:413-422.

34. Gilmer TP, O'Connor PJ, Rush WA, Crain AL, Whitebird RR, Hanson AM, Solberg LI. Impact of office systems and improvement strategies on costs of care for adults with diabetes. Diabetes Care 2006; 29:1242-1248.

35. Bernabei R, Landi F, Gambassi G, Sgadari A, Zuccala G, M or V, Rubenstein LZ, Carbonin P. Randomized trial of impact of integrated care and case management of older people living in the community. BMJ 1998; 316:23-27.

36. Llewellying-Jones RH, Baikie KA, Smithers H, Cohen J, Snowdon J, Tennant CC. Multifaceted shared care intervention for late life depression in residential care: randomised controlled trial. BMJ 1999; 319:676-682.

37. Simon GE, Von Korff M, Rutter C, Wagner E. Randomised trial of monitoring, feedback and management of care by telephone to improve treatment of depression in primary care. BM J 2000; 320:550-554.

38. Unutzer J, Katon W, Callahan CM, Williams JW Jr H unkeler $E, H$ arpole $L$, H offing $M$, Della Penna $R D$, Noël PH, Lin EH, Areán PA, Hegel $M T$, Tang $L$, Belin TR, Oishi S, Langston C; IM PACT Investigators. Improving Mood-Promoting Access to Collaborative Treatment. Collaborative care management of late-life depression in the primary care setting: a randomized controlled trial. JAM A 2002; 288:2836-2845.

39. Katon WJ, Von Korff M, Lin EH, Simon G, Ludman E, Russo J, Ciechanowski P, Walker E, Bush T. The Pathways Study: a randomized trial of collaborative care in patients with diabetes and depression. Arch. Gen. Psychiatry 2004; 61:1042-1049.

40. Wagner EH, Glasgow RE, Davis C, Bonomi AE, Provost $L, M$ cCulloch $D$, Carver $P$, Sixta C. Quality improvement in chronic illness care: a collaborative approach. Jt Comm J Qual Improv. 2001; 27:63-80.
41. Polonsky WH, Earles J, Smith S, Pease DJ, Macmillan M, Christensen R, Taylor T, Dickert J, Jackson RA. Integrating medical management with diabetes self-management training: a randomized control trial of the Diabetes Outpatient Intensive Treatment Program. Diabetes Care 2003; 26:3048-3053.

42. Vetter MJ, Bristow L, Ahrens J. A model for home care clinician and home health aide collaboration: diabetes care by nurse case management and community health workers. $\mathrm{H}$ ome $\mathrm{H}$ ealth C. Nurse 2004; 22:645-648.

43. Wagner EH, Davis C, Schaefer J, Von Korff M, Austin $B$. A survey of leading chronic disease management programs: are they consistent with the literature? M anag. Care Q. 1999; 7:56-66.

44. Malcom L, Wright L, Carson L. Integrating primary and secondary care: the case of Christchurch South Health Centre. N ew Zealand M ed. J. 2000; 113:514517.

45. M clnnes GT, M CGhee SM. Delivery of care for hypertension. J. Hum. H ypertens. 1995; 348:2635-2645.

46. Osman LM, Abdalla MI, Russell IT, Fiddes J, Friend JA, Legge JS, Douglas JG. Integrated care for asthma: matching care to the patient. Euro Respir J 1996; 9:444-448

47. Nuño RS. Un breve recorrido por la atención integrada. Revista de Innovación Sanitaria y Atención Integrada 2008; 1:2.

48. Rosen R, Ham C. Atención integrada: enseñanzas de evidencia y experiencia, informe del seminario Anual de Salud 2008 Sir Roger Banninster. Revista de Innovación Sanitaria y Atención Integrada 2008; 1:2.

49. Albuquerque $\mathrm{HC}$, Mendes EV. Las redes de atención a la salud en Janauba, M inas Gerais, Brasil: el caso de la red Viva Vida. Revista de Innovación Sanitaria y Atención Integrada 2009; 1:2.

50. M endes EV. Programa M ãe Curitibana: uma rede de atenção à mulher e à criança em Curitiba, Paraná. Lima: Organização Pan-Americana da Saúde; 2009.

Artigo apresentado em 06/04/2010

Aprovado em 27/05/2010

Versão final apresentada em 07/06/2010 